\title{
Biomimicking of the Breast Tumor Microenvironment
}

\author{
Marta Giussani ${ }^{1}$ - Carmelo De Maria ${ }^{2,3}$ - Vasso Michele ${ }^{4}$. \\ Francesca Montemurro $^{2} \cdot$ Tiziana Triulzi $^{1} \cdot$ Elda Tagliabue $^{1}$. \\ Cecilia Gelfi ${ }^{5,6,7} \cdot$ Giovanni Vozzig ${ }^{2,3}$
}

Published online: 26 April 2015

(C) Springer International Publishing AG 2015

\begin{abstract}
The tumor microenvironment is well known to play a role in sustaining malignant transformation of tissue, tumor progression, and in drug responsiveness; however, much remains unclear about the interplay between tumor cells, the extracellular matrix, and stroma cells. The extracellular matrix has been shown to elicit both biochemical and biophysical signaling, and matrix rigidity is an important microenvironmental parameter in the regulation of cellular behavior. Thus, tissue engineering and the development of novel biomaterials that mimic mechanical and topological properties of tumor stroma and can cope with the effect of mechanical forces are promising approaches to study this interplay. New in vitro
\end{abstract}

This article is part of the Topical Collection on Molecular Biology of Skeletal Tissue Engineering

Marta Giussani, Carmelo De Maria and Vasso Michele contributed equally to this work.

Giovanni Vozzig

g.vozzi@ing.unipi.it

1 Molecular Targeting Unit, Department of Experimental Oncology and Molecular Medicine, Fondazione IRCCS Istituto Nazionale dei Tumori, Milan, Italy

2 Research Center "E. Piaggio", University of Pisa, Pisa, Italy

3 Dipartimento di Ingegneria dell'Informazione (DII), University of Pisa, Pisa, Italy

4 Istituto di Bioimmagini e Fisiologia Molecolare (IBFM)-CNR, C.da Pietrapollastra-Pisciotto, Cefalù, 90015 Palermo, Italy

5 Dipartimento di Scienze Biomediche per la Salute, Università degli Studi Di Milano, Via F.lli Cervi 93, Segrate, 20090 Milan, Italy

6 IRCCS Policlinico San Donato, Piazza Edmondo Malan, San Donato Milanese, 20097 Milan, Italy

7 Istituto di Bioimmagini e Fisiologia Molecolare (IBFM)-CNR, Via F.lli Cervi 93, Segrate, 20090 Milan, Italy tools to investigate the effect of mechanical signals on breast cancer cell aggressiveness and drug sensitivity include genipin-crosslinked gelatin hydrogel scaffolds with adjustable degrees of stiffness.

Keywords Breast cancer · Extracellular matrix $\cdot 3 \mathrm{D}$ tumor tissue model $\cdot$ Mechanical characterisation $\cdot$ MALDI mass spectrometry $\cdot$ Glycans

\section{Introduction}

\section{Microenvironment and Cancer Progression}

In recent years, the role of the microenvironment in maintaining tissue specificity and organ structure and in promoting or inhibiting progression to malignancy has been widely recognized [ $1 \bullet]$. The mammary gland is a dynamic tissue composed of epithelial cells and surrounding stroma which not only modulates the normal development of the gland but also actively participates in its malignant transformation, contributing to tumor phenotype and disease progression. This suggests that the tumor tissue goes beyond the properties of the tumor epithelium itself and requires interconnections with the surrounding microenvironment $[2,3]$. The growing interest in deciphering the role of the tumor microenvironment in cancer progression is reflected by several recent studies based on gene expression profiling of tumor stroma [4-6]. For example, extensive gene expression changes have been observed in the stroma associated with ductal carcinoma in situ (DCIS) and invasive ductal carcinoma (IDC), suggesting the co-evolution of the tumor adjacent stroma with epithelium even before tumor invasion and supporting the important role of stromal changes in the transition from pre-invasive to invasive tumor growth [4]. Moreover, stromal characteristics have been 
shown to provide novel biological and clinically relevant insights into breast cancer progression [5,6]. These studies demonstrate that the tumor microenvironment is an important player in tumorigenesis. Analyses of expression patterns of genes encoding extracellular matrix (ECM) molecules have shown that stromal expression patterns can vary among breast carcinomas and may be clinically quite independent of the intrinsic characteristics of neoplastic cells [7, 8]. Recently, we identified an ECM gene expression signature (ECM3) in $\sim 40 \%$ of breast carcinomas that defines an independent group of tumors and has prognostic significance related to tumor differentiation status, stratifying a subgroup with poor prognosis only within the most undifferentiated grade III tumors $[9,10]$. The extracellular matrix is the major player of this niche composed of a large collection of biochemically distinct components including proteins, glycoproteins, proteoglycans, and polysaccharides with different physical and biochemical properties [11]. Communication between cells and the microenvironment occurs through a complex cascade of molecular signals generated by cell-matrix interactions as well as by the interplay between epithelial, stromal, and other organ-specific cell types (i.e., fibroblasts, adipocytes, myoepithelial cells, immune cells) $[1 \bullet]$. The cooperation between the mechanical microenvironment and the intrinsic cell state plays a key role in tumor progression through mechanical responsive sensors such as integrins, focal adhesion kinase, and cytoskeletal molecules able to elicit a specific cellular response [12, 13, 14•].

Alteration of ECM biochemical properties during microenvironment remodeling induced by changes in protein abundance and post-translational modifications potentiates the oncogenic effect of various signaling pathways (e.g., ERK, PI3K, TGF- $\beta$, and RhoA/Rac signaling), representing relevant cancer hallmarks [15]. In addition, the architecture and other physical characteristics of tumor-associated ECM may affect the properties of the stroma to change tissue rigidity. Epithelial cancers are characterized by an altered tissue tensional homeostasis that reflects the increment of cellgenerated forces in transformed cells, an increased compression due to the solid-state pressure exerted by the expanding tumor mass, and matrix stiffening associated with a desmoplastic response $[13,16]$. The malignant transformation of normal breast tissue is associated with significant matrix remodeling that triggers progressive stiffening [2]. Indeed, rigidity of breast cancer stroma is typically 10 -fold higher than that in normal tissue [17]. Models able to reproduce tumor complexity can provide tools for tumor-stroma interaction studies to pinpoint the molecular mechanisms involved in aggressiveness and resistance to therapy.

\section{Biological Tools Mimicking the Tumor Microenvironment}

Traditional in vitro culture platforms to study the role of the ECM in cancer have their limitations, and cancer biologists look with growing interest to the field of tissue engineering as a promising approach to obtaining "functional" in vitro tumors $[18,19]$ for a better understanding of chemical and mechanical interactions between tumors and the microenvironment [20]. One avenue is through the development of novel biomaterials that mimic biophysical, mechanical, and topologic properties of tumor stroma [21•]. Current approaches to tissue engineering have focused on hydrogel materials displaying ECM-like biophysical properties that provide dynamic microenvironments for cell fate regulation [18, 22]. Natural hydrogels are derived from or are themselves components of the ECM, such as Matrigel, collagen and fibrin scaffolds, whereas synthetic hydrogels are typically composed of polymers whose representative biomaterial is polyethylene glycol (PEG). Natural hydrogel have been widely used for 3D microenvironment mimicking to support tumor growth and angiogenesis, since they can be proteolytically degraded and remodeled by most cultured cell types [18]. Matrigel is a basement membrane-derived hydrogel containing laminin as the primary component. Extracted from Engelbreth-Holm-Swarm (EHS) mouse sarcoma cells, Matrigel is characterized by high cytocompatibility and cell adhesion sites and can change its physical properties over time [22, 23]. Collagen hydrogels, based on the most abundant ECM protein, are rich in cellinteractive ligands and thus able to reproduce a fibrous architecture similar to collagen structures of native ECM and to provide a bioactive microenvironment for cell culture; several different crosslinking methods are available to obtain different structures [19, 24, 25]. Fibrin hydrogels, obtained via polymerization of fibrinogen with thrombin and calcium ions,

Fig. 1 a Young modulus of gelatin-genepin scaffold as a function of gelatin percentage. Base peak chromatogram of glycans released after PNGase F treatment of b $2 \mathrm{kPa}, \mathbf{c} 15 \mathrm{kPa}$, and d $80 \mathrm{kPa}$ gelatin scaffold. PNGase F (Promega, Milano, Italy) digestion was carried out on the gelatin layer for $16 \mathrm{~h}$ at $37^{\circ} \mathrm{C}$ using $3 \mathrm{U} / \mu \mathrm{L}$ of enzyme in ammonium bicarbonate buffer ( $\mathrm{pH}$ 8.4). Released glycans were analyzed by LC-MS/MS. Mass spectra were recorded using an AmaZon Speed ETD mass spectrometer (Bruker Daltonics, Bremen, Germany) interfaced to an Easy-nLC chromatograph (Proxeon, Waltham, MA, USA). Glycans were separated by reverse-phase C18 chromatography and eluted on a 30-min ACN/0.1 \% formic acid (buffer B) gradient (Thermo, Waltham, MA, USA). e Representative areas of MDA-MB-231 and MCF7 cells cultured on 80 and $2 \mathrm{kPa}$ scaffolds in 24-well plates for $120 \mathrm{~h}$. Images were acquired with an optical microscope (Nikon Te-S) at $\times 10$ magnification (scale bar: $100 \mu \mathrm{m})$. f Histograms showing growth index of cells on scaffolds at different time points from cell seeding using the alamarBlue assay. At each time point, fluorescence intensity was read at $30 \mathrm{~min}$ and at $2.5 \mathrm{~h}$, when alamarBlue was added to the medium, using Tecan ULTRA Plate Reader XFLUOR4 version (MTX Lab Systems, Inc., VA, USA) at excitation $535 \mathrm{~nm}$ and emission $590 \mathrm{~nm}$. The 72-h time point was considered as reference to calculate the growth index. $\# 0.01<p<0.05$ compared with the $72 \mathrm{~h}$ condition; $* 0.01<p<0.05$ compared with the $80 \mathrm{kPa}$ scaffold at the 120 -h time point. Data are representative of one of three independent experiments in duplicate determinations. (Error bars $=\mathrm{SD})$ 

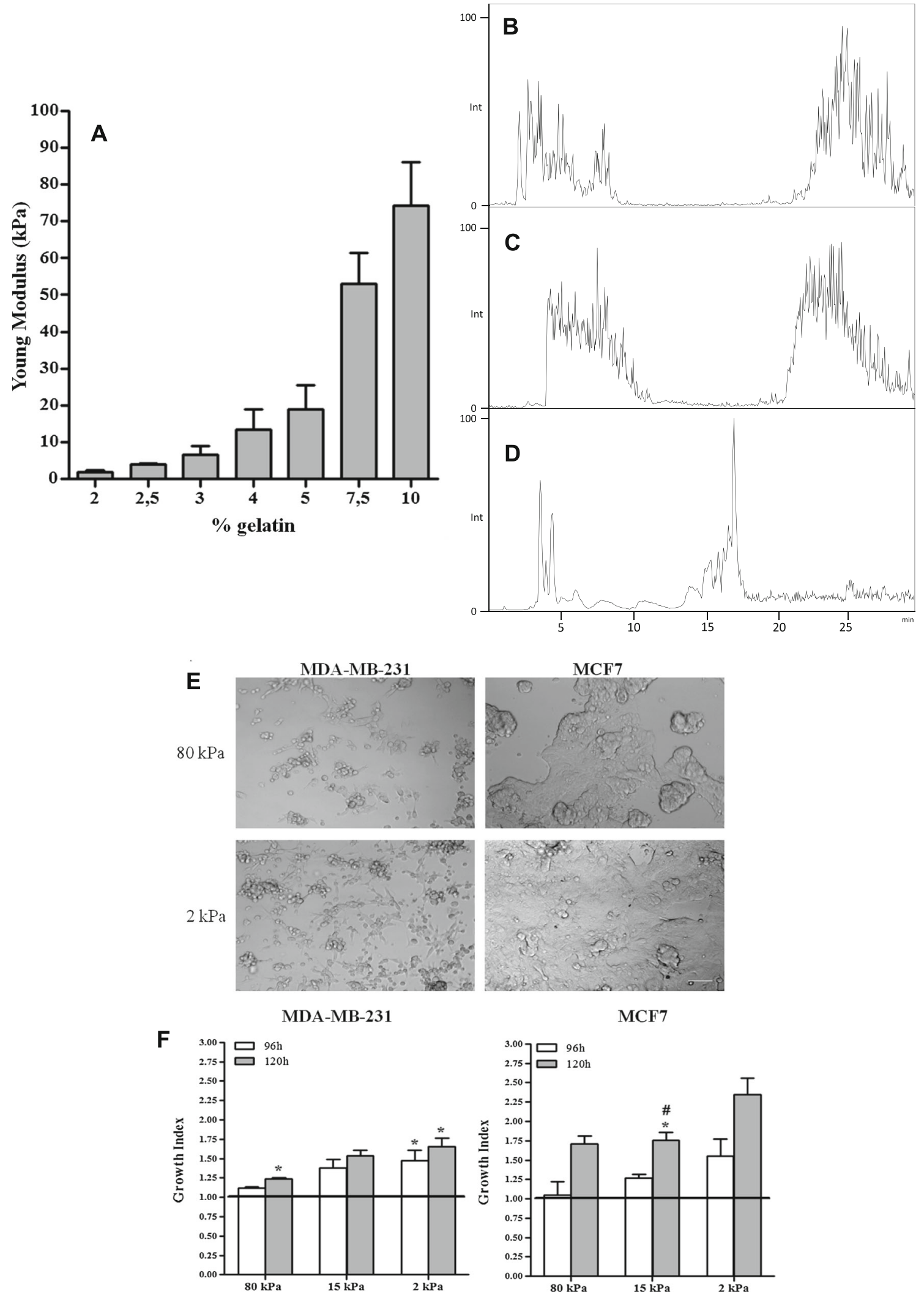

MCF7

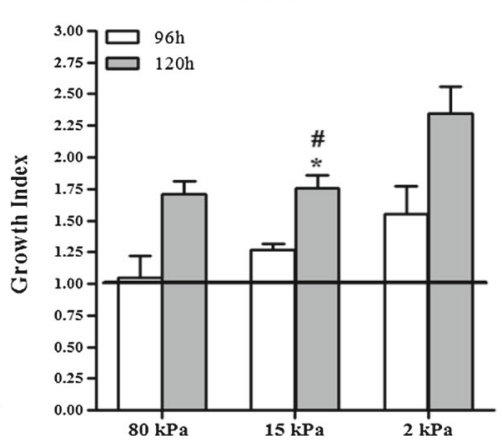


have also been widely used due to their nano/macro-fibrous architecture mimicking native ECM [26]. Increasing attention has focused on the development of synthetic hydrogels that present a time of proteolytic degradability, cell adhesion sites, and matrix stiffness similar to those during neo-tissue development; PEG-based hydrogel materials, characterized by a non-bioreactive structure coated with bioactive molecules (e.g., cell adhesion peptide, RGD sequence) or crosslinked with biopolymers (e.g., collagen, gelatin), fulfill those requirements [22]. Other hydrolytically degradable polymers in the polyester group, such as poly(lactic acid) or its poly(lactic-coglycolic acid) (PLG) copolymer, have also been widely used due to their non-cytotoxic degradation products and their tunable matrix mechanical properties [27•].

The ECM elicits not only biochemical but also biophysical signaling, and matrix stiffness is an important microenvironmental parameter that cooperates in regulating cell behavior both in promoting a malignant phenotype and in affecting response to therapy $[16,17,28,29]$. Thus, the development of new in vitro tools able to cope with the effect of mechanical forces is essential both to study tumor progression and to foster the discovery of anti-tumor drugs. To date, the most widely used substrates to generate scaffolds for assessment of the mechanical effects on tumor cells have been the flexible polyacrylamide substrates coated with collagen I [30-32], collagen I or collagen I and fibronectin [33, 34], collagen I hydrogels [19], and growth factor-reduced Matrigel [35]; however, those studies showed that the stiffness increment perturbs fibril topology and matrix pore size, introducing confounding biophysical cues and affecting the ECM natural topology. These side effects are relevant and affect the sensing on matrix organization, rendering them unsuitable for studies on breast carcinoma cells. Recently, a new self-assembling peptide hydrogel (SAP) has been described that recreates a native-like ECM fibril topology with minimal variability in pore size [36]. This hydrogel was able to influence the organization of mammary epithelial cell colonies with cell dissemination into the surrounding matrix; however, despite the high performance in recreating mechanical ECM properties, this system shows mechanical fragility, limiting its use for high-throughput analyses, e.g., for the screening of novel therapeutics designed to target tumor cells or modify tumor ECM.

\section{Genipin-Crosslinked Gelatin Hydrogel Scaffolds for Breast Cancer Cell Line Culture}

To reproduce the cell environment, a scaffold must mimic the biochemical, mechanical, and topological features of the ECM matrix. In recent years, efforts have focused on the use of natural polymers, principally based on ECM proteins and their derivatives, to produce scaffolds that mimic these ECM features. Tunable mechanical properties are especially important when developing tissue-specific scaffolds, where alterations in such features can be introduced by physical, enzymatic, or chemical crosslinking [37-39]. For example, gelatin is normally soluble in cell culture media but must be crosslinked to obtain a 3D self-consistent scaffold. To achieve this, different crosslinkers have been used, either synthetic such as glutaraldehyde or natural such as genipin, derived from gardenia fruit and non-toxic [40].

We have developed genipin-crosslinked gelatin hydrogel scaffolds to study their potential usefulness in analyzing the role of stiffness in the behavior of breast carcinoma cells according to their intrinsic molecular characteristics and in highthroughput assays to screen for new therapeutics. Genipin was chosen among other crosslinking agents to be sure of crosslinking reaction in the inner parts of the final samples, as indicated by blue coloring upon its spontaneous reaction with amino acids and proteins and because it is less toxic of other crosslinkers. The genipin-fixed scaffold resists enzymatic degradation at a level comparable to that of glutaraldehydefixed tissue, but genipin is less cytotoxic than glutaraldehyde $[41,42]$. These scaffolds were generated with stiffness values between 2 and $80 \mathrm{kPa}$ based on the typical value of 2-4 $\mathrm{kPa}$ reported for the Young acellular tumor extracellular matrix [43] and on the $16 \mathrm{kPa}$ to $130 \mathrm{kPa}$ increase in the elastic modulus with progression of invasive breast carcinoma $[44,45]$.

As shown in Fig. 1a, we are able to modulate the elastic modulus of scaffolds by changing the gelatin concentration, with increased stiffness upon increasing concentrations of gelatin, to mimic the tumor tissue microenvironment. In the context of structure characterization, the profile of exposed Nlinked oligosaccharides is a peculiar feature of genepin scaffolds with different gelatin concentrations $(2,4$, and $10 \%$ corresponding to 2,15 , and $80 \mathrm{kPa}$, respectively). Gelatin matrix crosslinked with genepin treated with PNGase F and analyzed by LC-MS/MS revealed a different profile of glycans, depending on the gelatin concentration (Fig. 1b-d). While the base peak chromatograms of cleaved oligosaccharides from 2- and $15-\mathrm{kPa}$ samples exhibited similar profiles, the $80-\mathrm{kPa}$ sample appeared different, suggesting that a more rigid support can induce changes in exposed glycans or that the glycan moiety plays an active role in matrix polymerization contributing to the polymer tridimensional structure and stiffness [46]. A better mechanistic understanding of the relationship between the bio-mimicked microenvironment and cell behavior awaits an in-depth characterization of the glycan surface exposed to protein interactions in scaffolds with different gelatin concentrations.

Evaluation of our hydrogel materials as new tools for cells in culture using two human cell lines representative of two breast cancer molecular intrinsic subtypes, i.e., MDA-MB231 for triple-negative and MCF7 for luminal A tumors, showed that both cell lines survived and grew on scaffolds 
with different stiffness (an elastic modulus of 2, 15, and $80 \mathrm{kPa}$ ), with significant and trend toward slowing proliferation as stiffness increased in MDA-MB-231 and MCF7, respectively (Fig. 1e, f). Furthermore, our genipin-crosslinked gelatin hydrogel scaffolds affected breast cancer cell behavior, inducing a more aggressive phenotype in both cell lines regardless of their intrinsic characteristics, according to their elastic modulus values. In MDA-MB-231 cells, a 2- to 3-fold increase in their migration potential was observed as a function of increased stiffness, while MCF7 cells, which are unable to migrate after culture in plastic plates, were induced to migrate when cultured on the stiffest scaffolds.

\section{Conclusions}

The established role of the tumor microenvironment in cancer progression, which mainly relies on biochemical properties, architecture, and other physical properties of tumor-associated ECM, has led to new approaches in tissue engineering to study the cancer-ECM relationship. The genipin-crosslinked gelatin hydrogel scaffold, which mimics not only biochemical but also mechanical forces of the tumor cell microenvironment, represents an available model in which it is possible to modulate stiffness. This scaffold could represent effective tools to investigate the effect of mechanical signals on tumor cell aggressiveness and drug sensitivity.

\section{Compliance with Ethics Guidelines}

Conflict of Interest Marta Giussani, Carmelo De Maria, Vasso Michele, Francesca Montemurro, Tiziana Triulzi, Elda Tagliabue, Cecilia Gelfi, and Giovanni Vozzi declare that they have no conflict of interest.

Human and Animal Rights and Informed Consent This article does not contain any studies with human or animal subjects performed by any of the authors.

\section{References}

Papers of particular interest, published recently, have been highlighted as:

- Of importance

1. Bissell MJ, Hines WC. Why don’t we get more cancer? A proposed role of the microenvironment in restraining cancer progression. Nat Med. 2011;17:320-9. Together with Chaudhuri et al. study this paper demonstrates the importance of extracellular tumor microenvironment on its progression.

2. Kass L, Erler JT, Dembo M, Weaver VM. Mammary epithelial cell: influence of extracellular matrix composition and organization during development and tumorigenesis. Int J Biochem Cell Biol. 2007;39:1987-94.

3. Pupa SM, Ménard S, Forti S, Tagliabue E. New insights into the role of extracellular matrix during tumor onset and progression. J Cell Physiol. 2002;192:259-67.

4. Ma XJ, Dahiya S, Richardson E, Erlander M, Sgroi DC. Gene expression profiling of the tumor microenvironment during breast cancer progression. Breast Cancer Res. 2009;11:R7.

5. Sharma M, Beck AH, Webster JA, Espinosa I, Montgomery K, Varma S, et al. Analysis of stromal signatures in the tumor microenvironment of ductal carcinoma in situ. Breast Cancer Res Treat. 2010;123:397-404.

6. Roman-Perez E, Casbas-Hernandez P, Pirone JR, Rein J, Carey LA, Lubet RA. Gene expression in extratumoral microenvironment predicts clinical outcome in breast cancer patients. Breast Cancer Res. 2012;19(14):R51.

7. West RB, Nuyten DS, Subramanian S, Nielsen TO, Corless CL, Rubin BP, et al. Determination of stromal signatures in breast carcinoma. PLoS Biol. 2005;3:e187.

8. Farmer P, Bonnefoi H, Anderle P, Cameron D, Wirapati P, Becette $\mathrm{V}$, et al. A stroma-related gene signature predicts resistance to neoadjuvant chemotherapy in breast cancer. Nat Med. 2009;15:68-74.

9. Bergamaschi A, Tagliabue E, Sorlie T, Naume B, Triulzi T, Orlandi $\mathrm{R}$, et al. Extracellular matrix signature identifies breast cancer subgroups with different clinical outcome. J Pathol. 2008;214:357-67.

10. Triulzi T, Casalini P, Sandri M, Ratti F, Carcangiu ML, Colombo MP, et al. Neoplastic and stromal cells contribute to an extracellular matrix gene expression profile defining a breast cancer subtype likely to progress. PLoS ONE. 2013;8: e56761. doi:10.1371/journal.pone.0056761.

11. Lu P, Weaver VM, Werb Z. The extracellular matrix: a dynamic niche in cancer progression. J Cell Biol. 2012;20(196):395-406.

12. Ng MR, Brugge JS. A stiff blow from the stroma: collagen crosslinking drives tumor progression. Cancer Cell. 2009;16: 455-7.

13. Butcher DT, Alliston T, Weaver VM. A tense situation: forcing tumour progression. Nat Rev Cancer. 2009;9:108-22.

14. Chaudhuri O, Koshy ST, da Branco CC, Shin JW, Verbeke CS, Allison $\mathrm{KH}$, et al. Extracellular matrix stiffness and composition jointly regulate the induction of malignant phenotypes in mammary epithelium. Nat Mater. 2014;13:970-8. Together with Bissel et al. study, this paper demonstrates how the properties of extracellular tumor microenvironment regulate tumor cell activities.

15. Pickup MW, Mouw JK, Weaver VM. The extracellular matrix modulates the hallmarks of cancer. EMBO Rep. 2014;15: 1243-53.

16. Provenzano PP, Inman DR, Eliceiri KW, Keely PJ. Matrix densityinduced mechanoregulation of breast cell phenotype, signaling and gene expression through a FAK-ERK linkage. Oncogene. 2009;28: 4326-43.

17. Levental KR, Yu H, Kass L, Lakins JN, Egeblad M, Erler JT, et al. Matrix crosslinking forces tumor progression by enhancing integrin signaling. Cell. 2009;139:891-906.

18. Gill BJ, West JL. Modeling the tumor extracellular matrix: tissue engineering tools repurposed towards new frontiers in cancer biology. J Biomech. 2014;47:1969-78.

19. Szot CS, Buchanan CF, Freeman JW, Rylander MN. 3D in vitro bioengineered tumors based on collagen I hydrogels. Biomaterials. 2011;32:7905-12.

20. Frantz C, Stewart KM, Weaver VM. The extracellular matrix at a glance. J Cell Sci. 2010;123:4195-200.

21. Schuessler TK, Chan XY, Chen HJ, Ji K, Park KM, Roshan-Ghias A, et al. Biomimetic tissue-engineered systems for advancing cancer research: NCI strategic workshop report. Cancer Res. 2014;74: 5359-63. An interesting paper on the application of hydrogels to reproduce cell tissue microenvironment. 
22. Song HH, Park KM, Gerecht S. Hydrogels to model 3D in vitro microenvironment of tumor vascularization. Adv Drug Deliv Rev 2014; 10 .

23. Benton G, Kleinman HK, George J, Arnaoutova I. Multiple uses of basement membrane-like matrix (BME/Matrigel) in vitro and in vivo with cancer cells. Int J Cancer. 2011;128:1751-7.

24. Szot CS, Buchanan CF, Freeman JW, Rylander MN. In vitro angiogenesis induced by tumor-endothelial cell co-culture in bilayered, collagen I hydrogel bioengineered tumors. Tissue Eng Part C Meth. 2013;19:864-74.

25. Fraker PJ, Speck JC. Protein and cell membrane iodinations with a sparingly soluble chloroamide, 1,3,4,6-tetrachloro 3-a, 6a diphenylglycoluril. Biochem Biophys Res Commun. 1978;80: 849-57.

26. Liu J, Tan Y, Zhang H, Zhang Y, Xu P, Chen J, et al. Soft fibrin gels promote selection and growth of tumorigenic cells. Nat Mater. 2012;11:734-41.

27. Drury JL, Mooney DJ. Hydrogels for tissue engineering: scaffold design variables and applications. Biomaterials. 2003;24:4337-51. An interesting paper on the application of hydrogels to reproduce the extracellular matrix of many tissues, and on their ability to be delivered in a minimally invasive manner.

28. Mouw JK, Yui Y, Damiano L, Bainer RO, Lakins JN, Acerbi I, et al. Tissue mechanics modulate microRNA-dependent PTEN expression to regulate malignant progression. Nat Med. 2014;20:360-7.

29. Bae YH, Mui KL, Hsu BY, Liu SL, Cretu A, Razinia Z. A FAKCas-Rac-lamellipodin signaling module transduces extracellular matrix stiffness into mechanosensitive cell cycling. Sci Signal. 2014;7:ra57.

30. Tilghman RW, Cowan CR, Mih JD, Koryakina Y, Gioeli D, SlackDavis JK, et al. Matrix rigidity regulates cancer cell growth and cellular phenotype. PLoS One. 2010;5:e12905.

31. Tilghman RW, Blais EM, Cowan CR, Sherman NE, Grigera PR, Jeffery ED, et al. Matrix rigidity regulates cancer cell growth by modulating cellular metabolism and protein synthesis. PLoS One. 2012;7:e37231.

32. Leight JL, Wozniak MA, Chen S, Lynch ML, Chen CS. Matrix rigidity regulates a switch between TGF-beta1-induced apoptosis and epithelial-mesenchymal transition. Mol Biol Cell. 2012;23: 781-91.

33. Menon S, Beningo KA. Cancer cell invasion is enhanced by applied mechanical stimulation. PLoS One. 2011;6:e17277.

34. Chen L, Xiao Z, Meng Y, Zhao Y, Han J, Su G, et al. The enhancement of cancer stem cell properties of MCF-7 cells in 3D collagen scaffolds for modeling of cancer and anti-cancer drugs. Biomaterials. 2012;33:1437-44.
35. Mi K, Wang G, Liu Z, Feng Z, Huang B, Zhao X. Influence of a self-assembling peptide, RADA16, compared with collagen I and Matrigel on the malignant phenotype of human breast-cancer cells in 3D cultures and in vivo. Macromol Biosci. 2009;9:437-43.

36. Miroshnikova YA, Jorgens DM, Spirio L, Auer M, SarangSieminski AL, Weaver VM. Engineering strategies to recapitulate epithelial morphogenesis within synthetic three-dimensional extracellular matrix with tunable mechanical properties. Phys Biol. 2011;8:026013-3975/8

37. Bat E, Kothman BH, Higuera GA, van Blitterswijk CA, Feijen J, Grijpma DW. Ultraviolet light crosslinking of poly(trimethylene carbonate) for elastomeric tissue engineering scaffolds. Biomaterials. 2010;31:8696-705.

38. Chen ZG, Wang PW, Wei B, Mo XM, Cui FZ. Electrospun collagen-chitosan nanofiber: a biomimetic extracellular matrix for endothelial cell and smooth muscle cell. Acta Biomater. 2010;6: 372-82.

39. Fathima NN, Dhathathreyan A, Ramasami T, Kragel J, Miller R. Degree of crosslinking of collagen at interfaces: adhesion and shear rheological indicators. Int J Biol Macromol. 2011;48:6773.

40. Zhang K, Qian Y, Wang H, Fan L, Huang C, Yin A, et al. Genipincrosslinked silk fibroin/hydroxybutyl chitosan nanofibrous scaffolds for tissue-engineering application. J Biomed Mater Res A. 2010;95:870-81.

41. Yao CH, Liu BS, Hsu SH, Chen YS, Tsai CC. Biocompatibility and biodegradation of a bone composite containing tricalcium phosphate and genipin crosslinked gelatin. J Biomed Mater Res A. 2004;69:709-17.

42. Bigi A, Cojazzi G, Panzavolta S, Roveri N, Rubini K. Stabilization of gelatin films by crosslinking with genipin. Biomaterials. 2002;23:4827-32

43. Lu WD, Zhang L, Wu CL, Liu ZG, Lei GY, Liu J. Development of an acellular tumor extracellular matrix as a three-dimensional scaffold for tumor engineering. PLoS One. 2014;9:e103672.

44. Au FW, Ghai S, Moshonov H, Kahn H, Brennan C, Dua H, et al. Diagnostic performance of quantitative shear wave elastography in the evaluation of solid breast masses: determination of the most discriminatory parameter. AJR Am J Roentgenol. 2014;203: W328-36.

45. Umemoto $\mathrm{T}$, Ueno $\mathrm{E}$, Matsumura $\mathrm{T}$, Yamakawa $\mathrm{M}$, Bando $\mathrm{H}$, Mitake T, et al. Ex vivo and in vivo assessment of the nonlinearity of elasticity properties of breast tissues for quantitative strain elastography. Ultrasound Med Biol. 2014;40:175568

46. Erler JT, Weaver VM. Three-dimensional context regulation of metastasis. Clin Exp Metastasis. 2009;26:35-49. 